\title{
Hemorragia Cerebral Intratumoral
}

\author{
Pedro Alessandro Leite de Oliveira* \\ Maria Paula Pelaiz** \\ Henrique Carrete Junior*** \\ João Norberto Stávale
}

\section{RESUMO}

As hemorragias cerebrais podem decorrer de tumores em cerca de $5 \%$ dos casos. Os tumores que mais sangram são: metástases (melanoma, coriocarcinoma, carcinoma de células renais), glioblastoma multiforme, oligodendroglioma e papiloma de plexo coróide. Apresentamos o caso de um paciente do sexo masculino de 69 anos com sintomas de início súbito, sendo identificada, pela tomografia de crânio, a presença de hematoma frontal direito. A análise dos aspectos radiológicos da lesão sugeriu tratar-se de hemorragia secundária a tumor cerebral, confirmada após craniotomia. O estudo anatomopatológico da lesão concluiu como glioblastoma multiforme com hematoma em organização. Nos casos de hemorragia cerebral deve-se pensar em etiologia neoplásica com base em dados clínicos e nos aspectos radiológicos da lesão.

\section{UNITERMOS}

Hemorragia intratumoral, hemorragia cerebral, glioblastoma multiforme.
Médico Residente da Disciplina de Neurologia da Escola Paulista de Medicina - Unifesp.

* Médica Residente do Departamento de Patologia Cirúrgica da Escola Paulista de Medicina - Unifesp.

*** Médico do Departamento de Diagnóstico por Imagem da Escola Paulista de Medicina Unifesp.

**** Professor-adjunto do Departamento de Patologia Cirúrgica da Escola Paulista de Medicina - Unifesp.

\section{INTRODUÇÃO}

Cerca de 5\% das hemorragias cerebrais decorrem de tumores intracranianos ${ }^{1}$. Na maioria dos casos, a apresentação clínica inicial é de instalação súbita, sugerindo quadro de acidente vascular cerebral hemorrágico de causa hipertensiva. É importante correlacionar aspectos clínicos e radiológicos para estabelecer uma causa tumoral. Neste artigo, apresentamos um caso ilustrativo que chegou ao nosso serviço com quadro ictal, cuja evolução clínica e os aspectos radiológicos evidenciaram como hematoma cerebral de natureza neoplásica devido a glioblastoma multiforme.

\section{RELATO DO CASO}

I.A.G., 69 anos, masculino, casado, aposentado, natural e procedente de São Paulo, SP.

Deu entrada no Pronto-socorro de Neurologia do Hospital São Paulo com queixa de diminuição da força muscular em membro superior esquerdo. $\mathrm{Na}$ noite anterior fora dormir bem e acordara no dia da admissão com fraqueza em membro superior esquerdo. Na tarde do mesmo dia, evoluiu com déficit de força em membro inferior esquerdo, sendo trazido ao nosso serviço. Negava sintomas neurológicos prévios. Com diagnóstico de hipertrofia prostática benigna, ex-tabagista, negava outras doenças prévias.

Ao exame físico geral de entrada, apresentava-se em regular estado geral, normocorado, com pressão arterial sistêmica de 140 X $90 \mathrm{mmHg}$, pulso de $80 \mathrm{bpm}$, com ritmo cardíaco regular, apresentando discreto sopro cardíaco pansistólico. Estava eupnéico, com ausculta pulmonar normal. Abdome plano, indolor à palpação, sem visceromegalias.

Ao exame neurológico, encontrava-se sonolento, porém, orientado no tempo e no espaço, obedecendo aos comandos verbais, com linguagem preservada. A avaliação dos nervos cranianos não revelou alterações, a fundoscopia ocular era normal. Trofismo e tônus muscular estavam normais. A força muscular era grau cinco em dimídio direito e grau três em dimídio 
esquerdo, com desvio de rima labial para a direita. Os reflexos estavam vivos à esquerda e normoativos à direita, com reflexo cutâneo plantar exibindo resposta em flexão à direita e em extensão à esquerda. As funções cerebelares e a sensibilidade estavam aparentemente normais.

Os exames de rotina da química sangüínea foram normais. Foi submetido à tomografia computadorizada de crânio sem contraste, a qual revelou imagem espontaneamente hiperdensa frontal direita, de aspecto heterogêneo, com discreto efeito expansivo e edema perilesional. Após injeção de contraste, observou-se discreto realce periférico incompleto. $\mathrm{O}$ aspecto heterogêneo associado aos realces e à localização não sugerem hemorragia espontânea, podendo corresponder a hematoma intratumoral (Figuras 1 e 2).

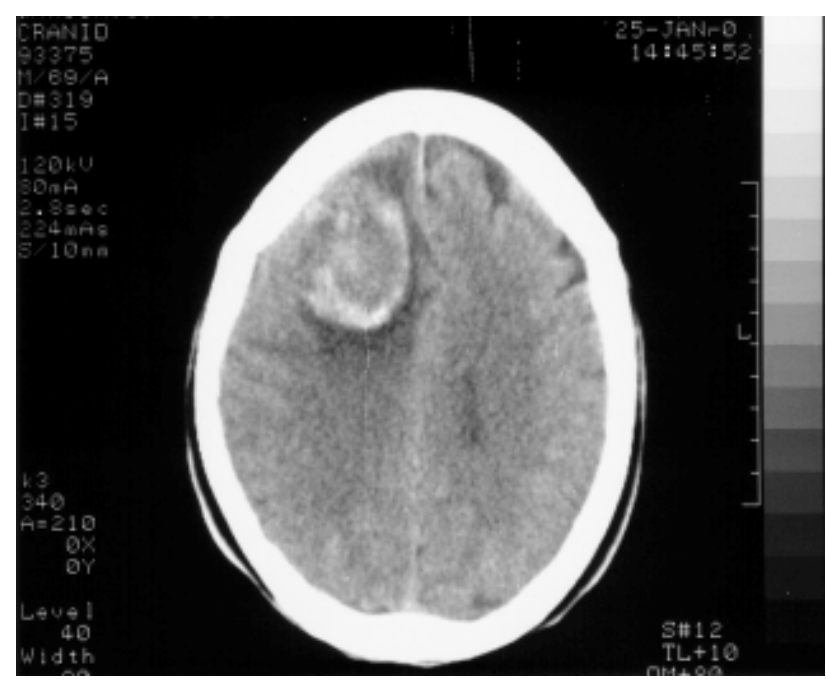

FIGURA1

Imagem de tomografia computadorizada de crânio, fase sem contraste, com imagem espontaneamente hiperdensa de aspecto heterogêneo, discreto edema e efeito de expansivo em região frontal direita.

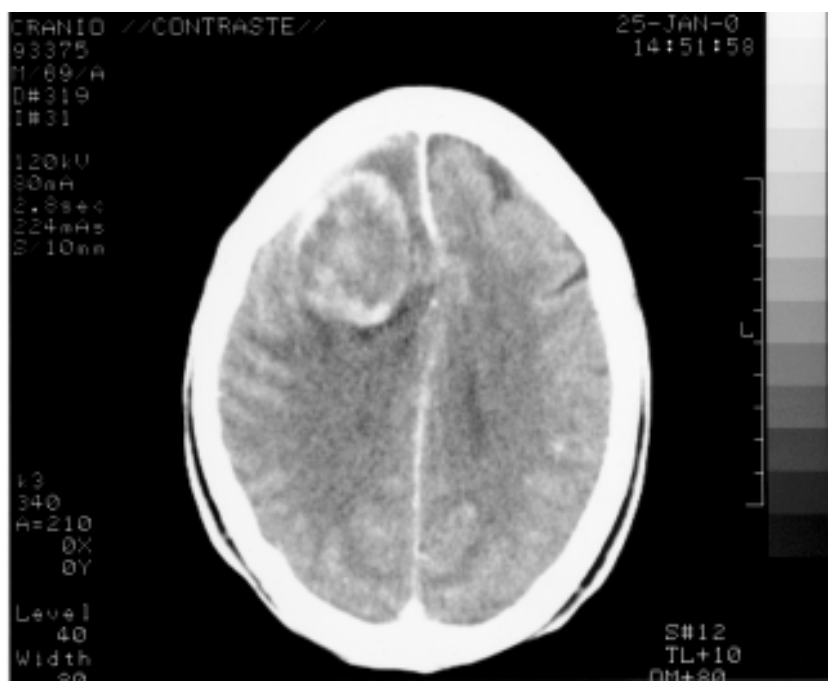

\section{FIGURA2}

Imagem de tomografia computadorizada de crânio, fase com contraste, com discreto realce periférico incompleto da lesão frontal direita. 
O paciente foi submetido à craniotomia frontal direita, evidenciando-se a presença de tumor cerebral com hematoma. $\mathrm{O}$ estudo anatomopatológico revelou tratarse de glioblastoma multiforme com hematoma em organização (Figuras 3A e 3B). Após esses resultados, o paciente foi tratado com quimio e radioterapia no Setor de Neurooncologia da Escola Paulista de Medicina Unifesp.
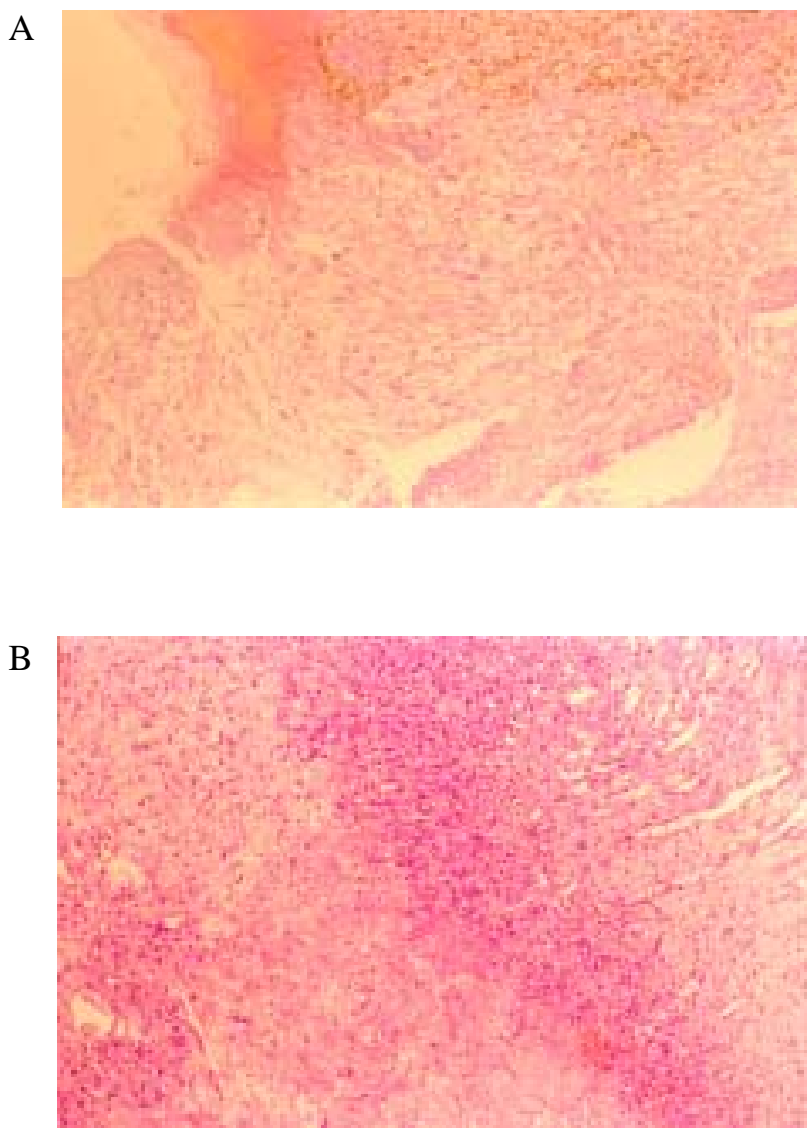

\section{FIGURA3}

A) Fotomicrografia revela glioblastoma multiforme caracterizado por células neoplásicas com acentuado pleomorfismo celular, hipercelularidade, citoplasma eosinofílico amplo, dispostas em meio a áreas de hemorragia recente e antiga (H \& E, 40x). B) Fotomicrografia revela glioblastoma multiforme onde se observa necrose "em paliçada" e endoteliose (H \& E, 40x).

\section{DISCUSSÃO}

A maioria dos tumores cerebrais manifesta-se por deterioração neurológica progressiva, porém, em alguns casos, a expressão clínica decorre de hemorragia no leito tumoral. As neoplasias cerebrais são responsáveis por cerca de $5 \%$ das hemorragias cerebrais ${ }^{1}$. Tais casos são mais freqüentemente descritos em tumores supratentoriais ${ }^{1}$. Na literatura, são raras as descrições de hemorragias em tumores infratentoriais; nesses casos os mais freqüentes são: o meduloblastoma, astrocitoma, ependimoma e metástase de melanoma ${ }^{2}$.

A tendência ao sangramento depende do subtipo histológico, ocorrendo com mais freqüência em tumores altamente vascularizados. A incidência de hemorragia é maior em tumores metastáticos que em tumores primários ${ }^{2-4}$. As neoplasias metastáticas que mais sangram são: o melanoma, coriocarcinoma e o carcinoma de células renais ${ }^{1}$. Dentre as neoplasias primárias, as que mais sangram são: o glioblastoma multiforme $(6,3 \%)$, o oligodendroglioma $(8,3 \%)$ e o papiloma de plexo coróide $(16,6 \%)^{1}$.

Há vários mecanismos patogenéticos aventados para explicar a hemorragia intratumoral. Descrevem-se seis possibilidades ${ }^{2}$ :

1) rotura de vasos malformados e finos do leito tumoral.

2) oclusão de vasos sagüíneos levando à proliferação endotelial anormal ou à embolia, acarretando infarto hemorrágico.

3) invasão da parede arterial pelo próprio tumor levando à rotura do vaso.

4) necrose vascular secundária à radioterapia.

5) alterações súbitas do gradiente de pressão intracraniana decorrentes da descompressão liquórica.

6) fatores metabólicos, em tumor de expansão rápida, restringindo a nutrição da porção central da lesão, provocando necrose e hemorragia.

No caso de tumores de crescimento rápido, como o glioblastoma multiforme, a taxa de expansão é maior na margem do tumor, sendo acompanhada de neoformação vascular nessa área. Assim, a parte central fica privada de nutrição adequada, levando à necrose e à hemorragia.

Em relação ao diagnóstico, nem sempre os achados são característicos o suficiente para facilitar o seu reconhecimento. É preciso avaliar os aspectos clínicos e radiológicos que sejam sugestivos de hemorragia tumoral. O quadro clínico caracteriza-se por instalação súbita ou subaguda, deteriorando em minutos ou em horas. Em alguns pacientes, há história prévia de deterioração neurológica progressiva seguida de piora aguda. Em outros, há um quadro ictal sem história prévia.

São descritos três padrões de achados tomográficos ${ }^{5}$ : 1) hematoma sólido: após contraste, pode-se observar realce tumoral na periferia do hematoma (mais comumente em metástases) ou realce contíguo ao hematoma. 
2) hemorragia central: decorre de rotura vascular no centro tumoral, mais freqüente em tumores da linguagem glial.

3) infarto hemorrágico: pode-se observar presença de sangue com formação de nível.

Outros dados que sugerem causa neoplásica para as hemorragias cerebrais: hemorragia em local atípico para etiologia hipertensiva ou aneurismática, ou que não corresponde a um território vascular cerebral definido, edema perilesional e realce após contraste ${ }^{5-7}$.

A ressonância magnética do crânio é importante por conseguir identificar tecido não hemorrágico dentro da hemorragia, sugerindo neoplasia.

O presente caso ilustra, portanto, a importância de se ter em conta que neoplasias podem ser a causa de hemorragias cerebrais e que tal suspeita deve ser levantada em casos nos quais o local de hemorragia, idade, aspecto radiológico e quadro clínico não forem compatíveis com hemorragias de natureza hipertensiva ou aneurismática. Deve-se identificar os tipos de imagem tomográfica do crânio que indicam a necessidade de estudo de neuroimagem com contraste.

\section{SUMMARY}

Tumoral Brain Hemorrage: Case Report

Cerebral hemorrhages may occurr in $5 \%$ of the cases of brain tumors. The most frequently bleeding tumors are: methastasis, gliobastoma, oligodendroglioma and choroid plexus papilloma. We report a man with 69 years of age who manifested a right frontal hemorrage with abrupt onset. Neuro-imaging studies suggested a tumoral secundary bleeding, which was surgically confirmed. Histhopathology showed a gliobastoma. Primary tumors of the brain must be included in the differential diagnosis of cerebra hemorrhage.

\section{KEYWORDS}

Tumoral brain hemorrhage, brain hemorrhage, glioblastoma.

\section{Referências}

1. Lieu AS, Huwang SL, Chai CY. Brain tumor with hemorrhage. J Formos Med Assoc, 98:365-7,1999.

2. Reichental $E$, Rubistein $A B$, Cohen ML. Infratentorial intratumoral hemorrhage. Mount Sinai J Med, 56:309-14,1989.

3. Black PM, Loeffler JS (eds.). Cancer of the nervous system. Neuropathology of Central Nervous System. 1 ed. Cambridge, Biacanrell Science, 1997, chap. 2, pp. 25-53.

Posner JB. Neurologic complications on cancer. 11 ed. Philadelphia, FA Devis Co, 1995, chap. 9, pp. 199-229.

5. Zimmerman RA, Bilariuk LT. Computed tomography of acute intratumoral hemorrhage. Radiology, 135:355-9,1980.

6. Meyer JR, Gorey MT. Differential diagnosis of non traumatic intracranial hemorrhage. Neuroimaging Clin N Am, 8:26393,1998.

7. Ogun AS, Olumale O, Ogunseyinde AO, Farade B, Odusote $\mathrm{KA}$. Misdiagnosed of stroke: a computerised tomography scan study. West Afr J Med, 19:19-22, 2000.

Endereço para correspondência:

Pedro Alessandro Leite de Oliveira

Disciplina de Neurologia

Escola Paulista de Medicina - Unifesp

Rua Botucatu, 740

CEP 04023-900 - São Paulo, SP

E-mail: pedmd99@yahoo.com.br 